\title{
Transverse profile imager for ultrabright electron beams
}

\author{
Rasmus Ischebeck, Eduard Prat, Vincent Thominet, and Cigdem Ozkan Loch \\ Paul Scherrer Institute, 5232 Villigen PSI, Switzerland \\ (Received 12 February 2015; published 27 August 2015)
}

\begin{abstract}
A transverse profile imager for ultrabright electron beams is presented, which overcomes resolution issues in present designs by observing the Scheimpflug imaging condition as well as the SnellDescartes law of refraction in the scintillating crystal. Coherent optical transition radiation emitted by highly compressed electron bunches on the surface of the crystal is directed away from the camera, allowing to use the monitor for profile measurements of electron bunches suitable for X-ray free electron lasers. The optical design has been verified by ray tracing simulations, and the angular dependency of the resolution has been verified experimentally. An instrument according to the presented design principles has been used in the SwissFEL Injector Test Facility, and different scintillator materials have been tested. Measurements in conjunction with a transverse deflecting radiofrequency structure and an array of quadrupole magnets demonstrate a normalized slice emittance of $25 \mathrm{~nm}$ in the core of a $30 \mathrm{fC}$ electron beam at a pulse length of $10 \mathrm{ps}$ and a particle energy of $230 \mathrm{MeV}$
\end{abstract}

DOI: 10.1103/PhysRevSTAB.18.082802

PACS numbers: 41.60.Cr, 41.60.Dk, 41.85.-p, 42.15.Eq

\section{INTRODUCTION}

$\mathrm{X}$-ray free electron lasers are becoming an indispensable tool to study molecular interactions on a femtosecond time scale $[1,2]$. To generate the $\mathrm{X}$-rays, ultrarelativistic electron beams are compressed and sent through a magnetic undulator [3]. The transverse size and shape of ultrabright electron beams are of great interest, hence transverse profile imagers that measure the particle distribution are key components of a free electron laser facility. A measurement of the electron profile is of particular interest for the determination of the beam area in transverse phase space, i.e., the transverse emittance. This parameter has a direct influence on the output power of a free electron laser. It also influences the gain length, which determines whether saturation of the FEL radiation can be achieved within the undulator length. Recent developments toward the highest brilliance X-rays and toward sub-femtosecond pulse length are leading to more stringent requirements on this instrumentation. Besides the increased demands on resolution, a new effect has been observed in recent years: highly compressed electron beams emit coherent optical transition radiation (COTR) at visible wavelengths [4-9], which impairs the measurement of profiles if it enters the imaging system.

Inorganic scintillators, which have been used in transverse beam profile measurements, help avoid this COTR [10,11].

"rasmus.ischebeck@psi.ch

Published by the American Physical Society under the terms of the Creative Commons Attribution 3.0 License. Further distribution of this work must maintain attribution to the author $(s)$ and the published article's title, journal citation, and DOI.
An additional benefit is that the emission of the scintillation light does not depend on the longitudinal structure of the bunches.

We present here a novel oblique imaging geometry, which fulfills three goals simultaneously for the first time: (i) The scintillator plane is imaged to the detector observing the Scheimpflug condition [12]; (ii) A scintillating crystal of finite thickness is imaged observing the Snell-Descartes law of refraction [13]; (iii) Coherent optical transition radiation is directed away from the camera axis. The first condition has been observed previously in an accelerator environment, both for imaging of scintillators [14] and optical transition radiation $[15,16]$. In the present implementation, the geometry is modified as explained in Sec. II. As a result, the monitor directs transition radiation away from the imaging system, yet allows for a high-resolution imaging of beams smaller than the thickness of the scintillating crystal. A transverse profile imager complying with these principles has been built and tested at the SwissFEL Injector Test Facility [17]. We report the time-resolved measurement of electron beam slice emittances of electron bunches with a total charge of only 30 femtocoulombs using a transverse deflecting mode rf cavity with one of these imaging stations.

An experimental verification of the COTR suppression is ongoing. First tests have been performed at LCLS [18], which hint at a suppression that is several orders of magnitude better than in standard LCLS monitors [16]. The presently installed monitor suffers from coherent diffraction radiation from the chamfer of the mirror, and further experiments and a comparison to wire scanner measurements are required to quantify the COTR suppression and the suitability for beam profile measurements. 


\section{IMAGING OF SCINTILLATING CRYSTALS}

\section{A. Observation geometries}

Profile monitors for the ionizing radiation typically consist of a scintillating crystal that can be inserted to intercept the primary beam inside a vacuum chamber, generating light that is imaged onto an image sensor. Excitation of the dopant levels in the scintillating crystal leads to an isotropic emission of light around the beam path in the medium. While different in their nature and in their applications, the effect of high-energy electron and photon beams on a scintillating crystal is very similar. As a consequence, most of the statements made in this paper are valid for both types of ionizing radiation, and differences will be explicitly stated. Figure 1 shows some commonly used layouts to image the fluorescent light onto the detector.

Each layout has specific limitations for the applicability at upcoming X-ray free electron lasers: Layout (a) may lead to radiation damage in the detector and is difficult to implement in an ultrahigh vacuum system. In layouts (b) $[19,20]$, (c) [11,21], and (d) [22] the horizontal resolution is limited to the scintillator thickness because points along the primary beam axis are imaged to different locations on the detector. The field of view is limited by the depth of field of the optical system. Both issues are exacerbated for magnifying optics. Application of the Scheimpflug principle [12,23], shown in layout (d), improves the field of view, but the limitation due to the finite scintillator thickness remains. Layouts (e) [24] and (f) [25] can be set up to generate excellent resolution, smaller than the thickness of the scintillating crystal. However, the mirror intercepts the primary beam, which may lead to a gradual loss of reflectivity due to radiation damage, and the scattered particles may pose radiation protection issues.

Optical transition radiation, generated by charged particle beams at vacuum-scintillator and vacuum-mirror interfaces, is emitted toward the camera in layouts (a), (b), (d), and (e). The distribution of a microbunched beam that emits fully coherent radiation may be determined from

(a)

(b)

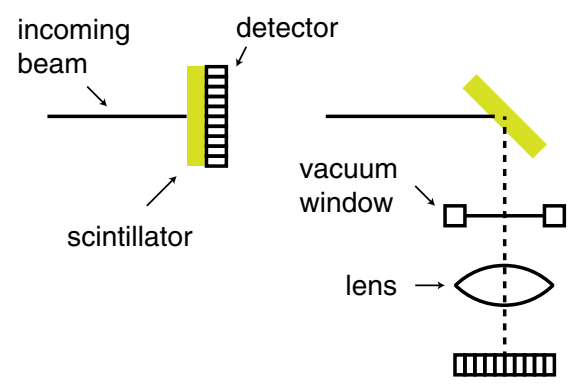

(c)

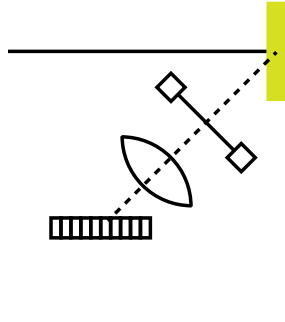

phase retrieval of the far-field radiation [26]. However, for a partially coherent emission of radiation with random transverse spatial locations of the intense COTR, the measurement of beam profiles with incoherent OTR is problematic at best [27]. The intense coherent light can be suppressed temporally by gating the exposure of the camera to start after the emission of the prompt transition radiation, and to integrate only the delayed scintillation light [28]. This method requires a shutter which opens on a nanosecond time scale, and which effectively suppresses the coherent radiation which may be several orders of magnitude more intense. To the authors' knowledge, this is currently only possible with intensified cameras that use microchannel plates, which add a significant layer of complexity, and which have limited lifetime [29].

A geometric suppression, as proposed in the present layout, is independent of the camera readout, and would thus not be impaired by beam repetition rates that are higher than the camera frame rate, such as in superconducting accelerators like the European XFEL [30] and LCLS-II [31].

\section{B. Choosing an observation angle according to the Snell-Descartes law of refraction}

A beam of ionizing radiation generates a scintillating column along its axis through the entire depth of the crystal. The breadth of this scintillating column depends on the properties of the scintillator. Screens made from powdered scintillators show significant broadening due to light scattering within the screen volume [32]. Scintillating crystals are compared in [33].

It is assumed here that the primary beam passes the crystal undeflected, an assumption that is well fulfilled for high energy beams used in X-ray free electron lasers, and that the emission of fluorescent light is isotropic. A beam with zero transverse size generates a scintillating column with a length given by the thickness $d$ of the scintillator and the angle of incidence $\alpha$. This column is imaged onto a single point on the detector only for two observation

FIG. 1. Commonly used layouts to image fluorescent light onto a detector. (a) Direct detection of the light, (b) and (c) imaging the light through a vacuum window, (d) observing the Scheimpflug imaging geometry, (e) using an additional in-vacuum mirror, and (f) with an additional mask to block coherent transition radiation. 

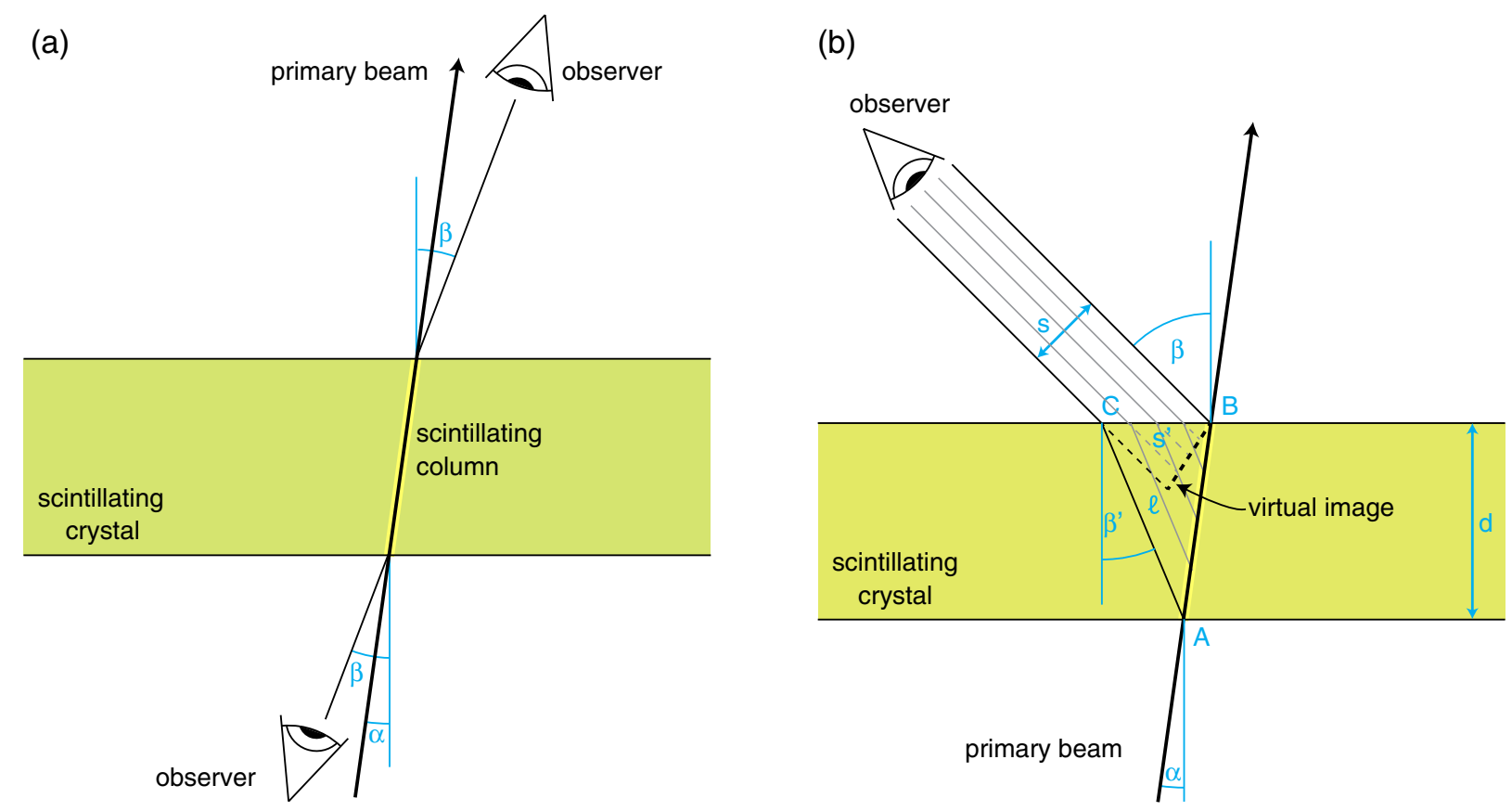

FIG. 2. Observation of light from a scintillating crystal. (a) Ideal observation angle, observing the Snell-Descartes law of refraction. (b) Situation for an arbitrary observation angle $\beta$.

angles, dictated by the Snell-Descartes Law of refraction [13], shown in Fig. 2(a). We assume an index of refraction $n$ in the crystal, surrounded by vacuum. If one chooses an observation angle $\beta$ such that $\sin \alpha=n \sin \beta$ then the scintillating light emanating from the primary beam axis is imaged onto the same point, assuming that the depth of field of the imaging system is larger than the crystal thickness [34].

\section{Imaging at arbitrary observation angles}

We will now look at the expected resolution when choosing an arbitrary observation angle. Figure 2(b) shows the imaging geometry for this case. Here, the primary beam enters a scintillating crystal of thickness $d$ at an angle $\alpha$ to the normal. The primary beam produces a uniformly radiating slab of length $d / \cos \alpha$, which is observed at a distance much larger than $d$, under an angle $\beta$ to the normal. Because of refraction on the surface of the crystal, the observer sees a virtual image of this slab, shown by a dashed line in Fig. 2(b).

We now look at the observation geometry in detail. Initially, we consider only the chief rays to an observer located at infinity, i.e., the observation with parallel rays. From the Snell-Descartes law of refraction [13], we get

$$
\frac{\sin \beta}{n}=\sin \beta^{\prime}
$$

Furthermore, the length of $\ell:=\overline{A B}$ can be calculated as:

$$
\ell:=\overline{A B}=\frac{d}{\cos \beta^{\prime}}
$$

and the length of $s^{\prime}:=\overline{B C}$ is

$$
s^{\prime}:=\overline{B C}=\frac{s}{\cos \beta} .
$$

The cosine theorem of the triangle $A B C$ yields to:

$$
s^{\prime 2}=\ell^{2}+\left(\frac{d}{\cos \alpha}\right)^{2}-2 \ell \frac{d}{\cos \alpha} \cos \left(\alpha+\beta^{\prime}\right) .
$$

From all of the above, the transverse size $s$ of this virtual image can be derived:

$$
s=d \cos \beta \cdot \sqrt{\frac{1}{1-\frac{\sin ^{2} \beta}{n^{2}}}+\frac{1}{\cos ^{2} \alpha}-2 \frac{\cos \left[\arcsin \left(\frac{\sin \beta}{n}\right)+\alpha\right]}{\sqrt{1-\frac{\sin ^{2} \beta}{n^{2}}} \cos \alpha}} .
$$

This apparent size is zero $(s=0)$ exactly when:

$$
\beta_{\text {ideal }}=-\arcsin (n \sin \alpha) .
$$

We thus recognize the Snell-Descartes law of refraction, the ideal observation angle shown in Fig. 2(a).

This result can be generalized to an observer located at a finite distance, imaging the object with a finite entrance pupil. The refraction at the surface of the crystal makes the virtual image appear closer to the observer, as shown in Fig. 2(b). The ideal focusing point of the camera has to be shifted by a fraction of the scintillator thickness. If we assume that the diameter of the entrance pupil of the lens is 
much larger than the field of view, the observation angle is the same for the entire extent of the beam, and the apparent size is not changed.

\section{Simulation with ray tracing}

These findings are confirmed by a ray tracing simulation of the imaging geometry, similarly to the work presented in $[28,33,35]$. For the present situation, the imaging of a YAG scintillator was modeled using Zemax OpticStudio [36]. The following parameters were modeled: a primary beam with zero transverse size is intercepted by a scintillating crystal, which has a thickness of $100 \mu \mathrm{m}$ and a refractive index of 1.8632 at a wavelength of $550 \mathrm{~nm}$. The primary beam has an incidence angle of $8.1^{\circ}$ to the scintillator normal, and the emission is simulated by five field points at depths of $[0,25,50,75,100] \mu \mathrm{m}$ along the beam. This light is imaged with a perfect lens of $f=200 \mathrm{~mm}$, and an aperture of $50 \mathrm{~mm}$, corresponding to $f / 4$. The lens is located at a distance of $400 \mathrm{~mm}$ to the scintillator, such that the magnification is $M=-1$. For this magnification, the effective $F$-number of the lens is 8 , and the corresponding numerical aperture is $N A=0.0625$. The simulation is performed at various observation angles $\beta$.

The simulated spot size consists of three components: (i) the shift between the first and the last point at 0 and $100 \mu \mathrm{m}$ depth, respectively, (ii) the defocus due to the depth of field, and (iii) the diffraction at the lens aperture, which can be calculated as $d=\lambda / 2 \sin \vartheta$ with a halfopening angle $\vartheta$.

The first contribution depends on the observation angle, and agrees with the analytical expression (1), as shown in Fig. 3. It is smallest for an observation angle of $15^{\circ}$, as predicted by Eq. (2). For this situation, the defocus amounts to $3.6 \mu \mathrm{m}$, and the diffraction limit of the lens results in an Airy disk of $5.38 \mu \mathrm{m}$ radius. A real lens would add

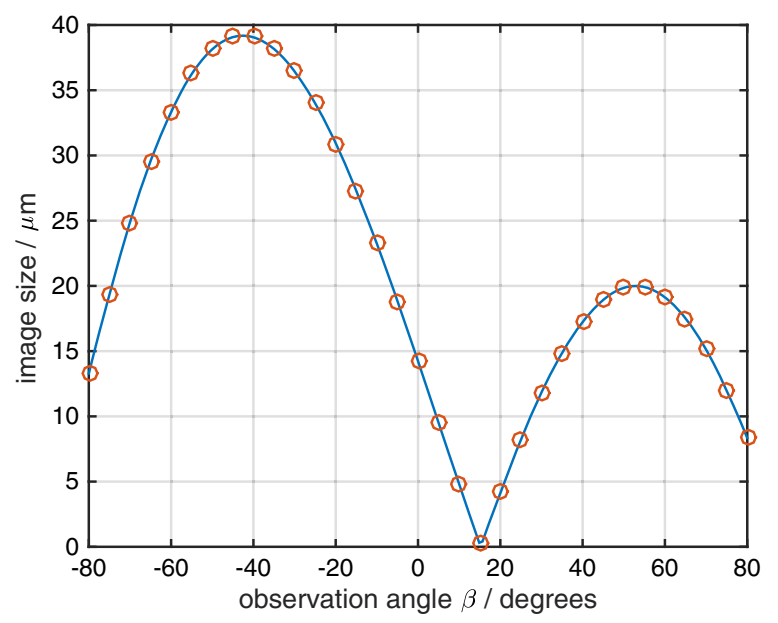

FIG. 3. Ray tracing results of the image size (red circles) agree with the analytical expression (1) (blue line). additional aberrations as compared to an ideal lens. Measurements of the point spread function (PSF) show an overall resolution of $8 \mu \mathrm{m}$ rms [18].

\section{E. Experimental verification of angle dependency}

The angular dependency of the imaged spot size was verified experimentally. Scintillation light was generated by $3 \mathrm{eV}$ photons from a diode laser $(\lambda=410 \mathrm{~nm})$, focused to a spot of $w_{0}=45 \mu \mathrm{m}$ FWHM into a $500 \mu \mathrm{m}$ thick Ce:YAG scintillator. The Rayleigh length is $L_{R}=\pi w_{0}^{2} / \lambda=16 \mathrm{~mm}$, thus the beam diameter can be considered uniform within the crystal. The green scintillation light was imaged onto a CCD detector with magnification $M=-1$, excluding the exciting light with a longpass filter. The full width at half maximum (FWHM) of the projected beam profiles is shown in Fig. 4. for an angle $\alpha=24^{\circ}$ of the scintillating column.

\section{F. Possible observation geometries}

For $\alpha=0$, the ideal observation angle is $\beta=0$, corresponding to the rectilinear observation geometry (e) in Fig. 1. This layout is used for the low-energy screens in the SwissFEL Injector Test Facility. Beamlets of a size of $14 \mu \mathrm{m}$ rms have been measured with $200 \mu \mathrm{m}$ thick scintillating crystals [37].

Choosing an oblique angle $\alpha \neq 0$ however offers unique opportunities for electron and X-ray beams, respectively: (i) For electron beams, the effect of camera saturation through coherent optical transition radiation emitted from the scintillator surface may be avoided by directing the radiation away from the optics. (ii) For X-ray beams above about $5 \mathrm{keV}$, a semitransparent monitor may be built if one chooses a suitable low-Z scintillator such as diamond [21], because the radiation can be observed through an off-axis mirror.

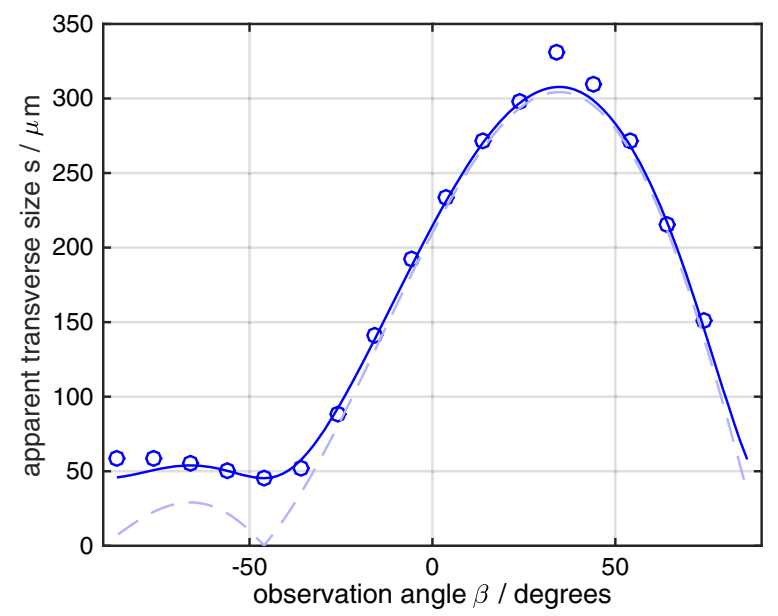

FIG. 4. Observed beam size, as a function of observation angle $\beta$. Circles: experimentally obtained data, dashed line: calculated values using Eq. (1), solid line: calculated values, with the initial beam size added in quadrature. 
The discussion up to this point allows selection of an ideal observation angle for arbitrary beam angles between zero and $\arcsin (1 / n)$. There is, however, an additional constraint, which is based on the detector. The angle between the incident photons and the detector normal depends on the magnification $M$ of the lens, and is given by the Scheimpflug criterion $\arctan (\tan \beta / M)$. Most commercially available detectors employ microlens arrays to increase the efficacy of light collection onto the sensitive elements on the detector surface. This technology limits the acceptance angle of the detector to a cone of typically $15^{\circ}$ to $20^{\circ}$ around the detector normal. As a consequence, a larger observation angle limits the choice of detector to those that can be obtained without a microlens array.

\section{TRANSVERSE PROFILE MONITOR}

\section{A. Construction of the monitor}

A transverse profile imager built to observe the SnellDescartes law of refraction as well as the Scheimpflug imaging criterion has been installed at the end of the SwissFEL Injector Test Facility [17] at Paul Scherrer Institut, Switzerland. The SwissFEL Injector Test Facility was built to demonstrate the generation of electron beams suitable for the upcoming X-ray free electron laser SwissFEL [38]. The goal is to generate beams with a core slice emittance of $430 \mathrm{~nm}$ at a charge of $200 \mathrm{pC}$, and of $180 \mathrm{~nm}$ at a charge of $10 \mathrm{pC}$. The electrons are generated by a UV laser pulse in a radio frequency (rf) photocathode gun. The particle bunches are accelerated by rf cavities to an energy of up to $250 \mathrm{MeV}$ per particle.

Four scintillators are installed: a cerium doped yttrium aluminum garnet crystal (Ce:YAG) [39], a nitrogen doped synthetic diamond multicrystal [40], a mixed rare-earth silicate crystal "CRY019" [41], and a chromium-doped aluminum oxide ceramic "CHROMOX" [42]. The thickness of the scintillating crystals is $100 \mu \mathrm{m}$, and they are polished on both sides. A conductive layer of indium tin oxide (ITO) has been applied to avoid the collection of surface charge on the Ce:YAG and CRY019 crystals. The ceramic screen has a thickness of $1 \mathrm{~mm}$.

The scintillators have a diameter of $20 \mathrm{~mm}$, but the field of view is horizontally constrained by the in-vacuum mirror [43] to $\pm 3 \mathrm{~mm}$. This is sufficient for the present application. A variant of the screen monitor with a larger field of view has been built by using an observation angle of $30^{\circ}$.

The screens are imaged by an $f=200 \mathrm{~mm}$ macro lens [44] to a CMOS detector [45]. The magnification of the imaging system is $1: 1.22$, resulting in an effective pixel size on the scintillator of $7.9 \mu \mathrm{m}$.

The scintillators are positioned at an angle of $8.1^{\circ}$ to the primary beam, and the in-vacuum mirror is placed such that the normal of the crystal has an angle of $15^{\circ}$ to the optical axis of the camera. The geometry is shown in Fig. 5. To fulfill the Scheimpflug imaging condition, the normal of

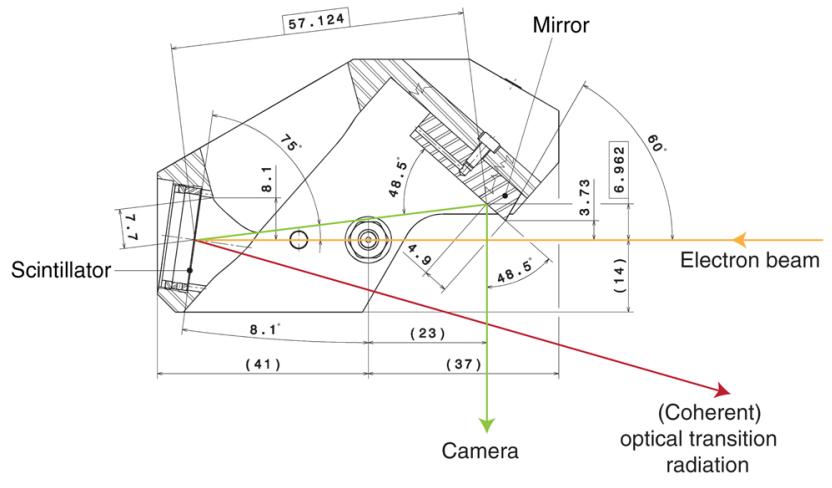

FIG. 5. Cross section of the screen mount, with the electron beam coming from the right. The angle from the normal of the scintillator is $8.1^{\circ}$ to the beam axis, and $15^{\circ}$ to the optical axis of the camera.

the CMOS detector is tilted by $14^{\circ}$ to the optical axis. At this angle, the microlens array on the chip can be used with only minor loss in efficiency.

The screens are mounted to an aluminum frame. They can be positioned into the beam by means of an ultrahigh vacuum translation stage. An in-vacuum mirror allowed the positioning of the camera at an observation angle of $90^{\circ}$ to the beam axis, which permitted the use of a compact vacuum chamber of $137 \mathrm{~mm}$ length. The field of view is $6 \times 14 \mathrm{~mm}^{2}$.

For reference measurements, optical transition radiation (OTR) was generated by charged beams on a $5 \mu \mathrm{m}$ thick silicon wafer [46]. The emission of OTR is a highly directional cone with an opening angle of $1 / \gamma$, centered around an axis opposite to the normal of the surface. In the present monitor, the silicon wafer was installed at an angle of $15^{\circ}$ to the beam axis. The transition radiation was reflected into the camera with an additional in-vacuum mirror, which has been positioned such that the imaging condition was the same as for the scintillator.

To increase the dynamic range of the profile imager, two neutral density filters [47] with $10 \%$ and $1 \%$ transmission, respectively, could be inserted into the optical path.

\section{B. Measurements of resolution and slice emittance}

The resolution of these scintillators was compared by focusing the electron beam horizontally onto each screen. The beam size was determined by fitting a Gaussian function to the projection of the image. Table I lists the values. For these measurements, the beam was not compressed, and as such no coherent optical transition radiation is expected. The resulting rms beam sizes can thus be compared to an OTR measurement.

Optical transition radiation is emitted in a cone around the beam axis, and the resolution that can be achieved with a perfect lens can be calculated analytically [48]. If the present $f=200 \mathrm{~mm}$ lens were replaced by a perfect lens 
TABLE I. Measured beam image size on different scintillators, and compared to optical transition radiation.

\begin{tabular}{lcc}
\hline \hline Scintillator & $\begin{array}{c}\text { Observed rms } \\
\text { beam image } \\
\text { size }[\mu \mathrm{m}]\end{array}$ & $\begin{array}{c}\text { Light yield } \\
\text { relative } \\
\text { to OTR }\end{array}$ \\
\hline YAG & 16.4 & 252 \\
CRY019 & 23.4 & 102 \\
Diamond & 106.6 & 1.9 \\
CHROMOX & 252.2 & 432 \\
OTR (for comparison) & 15.8 & 1 \\
\hline \hline
\end{tabular}

with the same aperture, we would expect a ringlike point spread function on the detector plane, with a radius of $3.5 \mu \mathrm{m}$ and a FWHM of $11.2 \mu \mathrm{m}$. The observed beam size is about a factor of five larger.

The broadening for Ce:YAG, observed in Ref. [33], could not be confirmed in the present measurements, where image sizes of $1 \sigma=16.4 \mu \mathrm{m}$ have been observed. This could be mostly due to the thinner scintillator in the present experiment.

A transverse deflecting rf structure is installed after the accelerating structures, allowing the vertical streaking of the electron beam and thus converting the vertical spatial axis to a time axis on the screen. In conjunction with an electron beam optics consisting of adjustable quadrupole lenses and the transverse profile imager, measurements of the transverse phase space of the beam have been performed. This includes a measurement of the slice emittance of the beam, i.e., the time-resolved measurement of the transverse phase space volume. The measurement method is illustrated in Fig. 6.

Five quadrupole magnets are used to generate the electron beam optics required for the slice emittance measurement: (i) The horizontal phase-advance is regularly scanned to cover a total of about 150 degrees to precisely reconstruct the horizontal emittance. (ii) The vertical phase-advance between the transverse deflector and the profile monitor is fixed to about 90 degrees to optimize the longitudinal resolution of the transverse deflector: with a maximum deflecting voltage of $5 \mathrm{MV}$ and a $\beta$-function at the transverse deflector of $40 \mathrm{~m}$, the longitudinal resolution is about 13 fs (assuming a projected vertical emittance of $500 \mathrm{~nm}$ and a beam energy of $250 \mathrm{MeV}$ ). (iii) The horizontal $\beta$-function is kept relatively high (between $35 \mathrm{~m}$ and $40 \mathrm{~m}$ ) to have beam sizes that can be conveniently measured even for very low emittances. Considering a beam size resolution of $15 \mu \mathrm{m}$ and assuming a beam energy of $250 \mathrm{MeV}$, for a $\beta$-function of $35 \mathrm{~m}$ the emittance resolution is about $3 \mathrm{~nm}$. (iv) The vertical $\beta$-function is kept below $10 \mathrm{~m}$ to control the beam size in the streaked direction.

At each optics the phase of the transverse deflector is varied to obtain individual $y-t$ calibrations. The beam was split into slices using the centroid from a Gaussian fit as a reference, and the beam size was obtained for each slice. From the beam sizes at each optics the slice emittances along the bunch are obtained. The measurement and data analysis are described in more detail in [49].

Figure 7 shows two examples of a slice emittance measurement of a beam with a total charge of $30 \mathrm{fC}$ and $10 \mathrm{pC}$, respectively. The normalized slice emittance for the $30 \mathrm{fC}$ case is below $25 \mathrm{~nm}$. The error bars indicated in the plots correspond to the statistical uncertainties calculated from the statistical errors of the beam size measurements. The calibration error of the profile monitor is of the order of $1 \%$, which causes a systematic error of the reconstructed emittances of around $2 \%$. The calibration uncertainty is

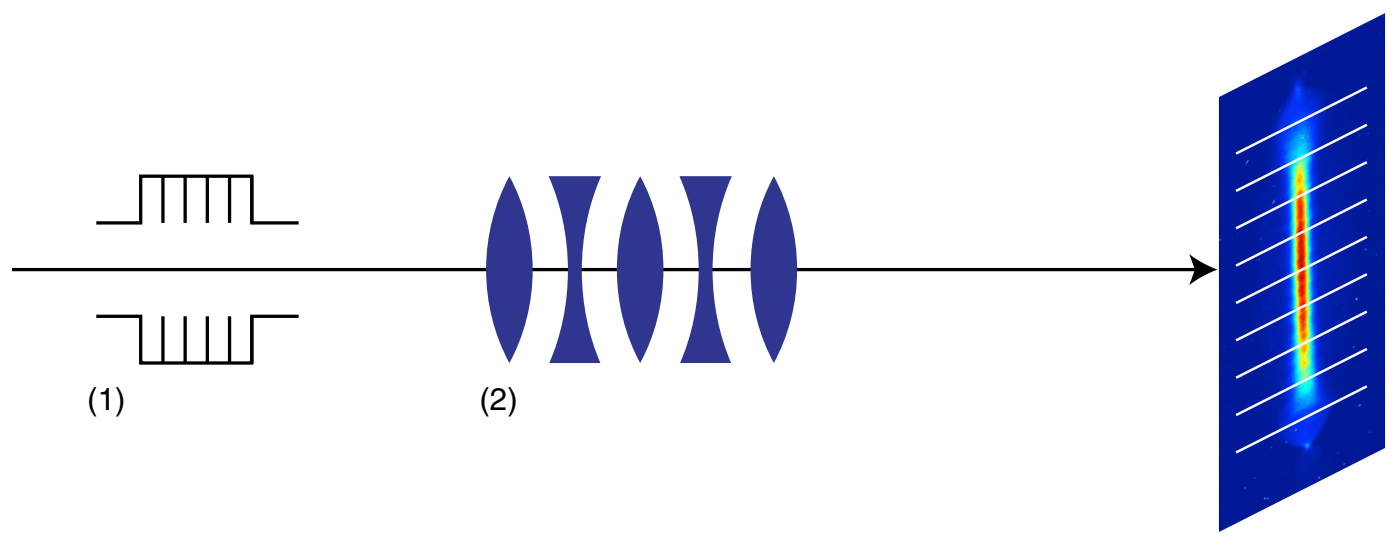

(3)

FIG. 6. Illustration of slice emittance measurements in a particle accelerator. The beam enters from the left and undergoes the following transformations: (1) mapping of the time axis onto the vertical angle by the transverse deflecting structure, (2) variation of the horizontal phase advance between the deflector and the profile monitor by adjusting the quadrupole lenses, while keeping the vertical phase advance approximately constant such that a vertical angle is transformed optimally into a vertical position, and (3) measurement of the horizontal beam size in several slices of the beam. 

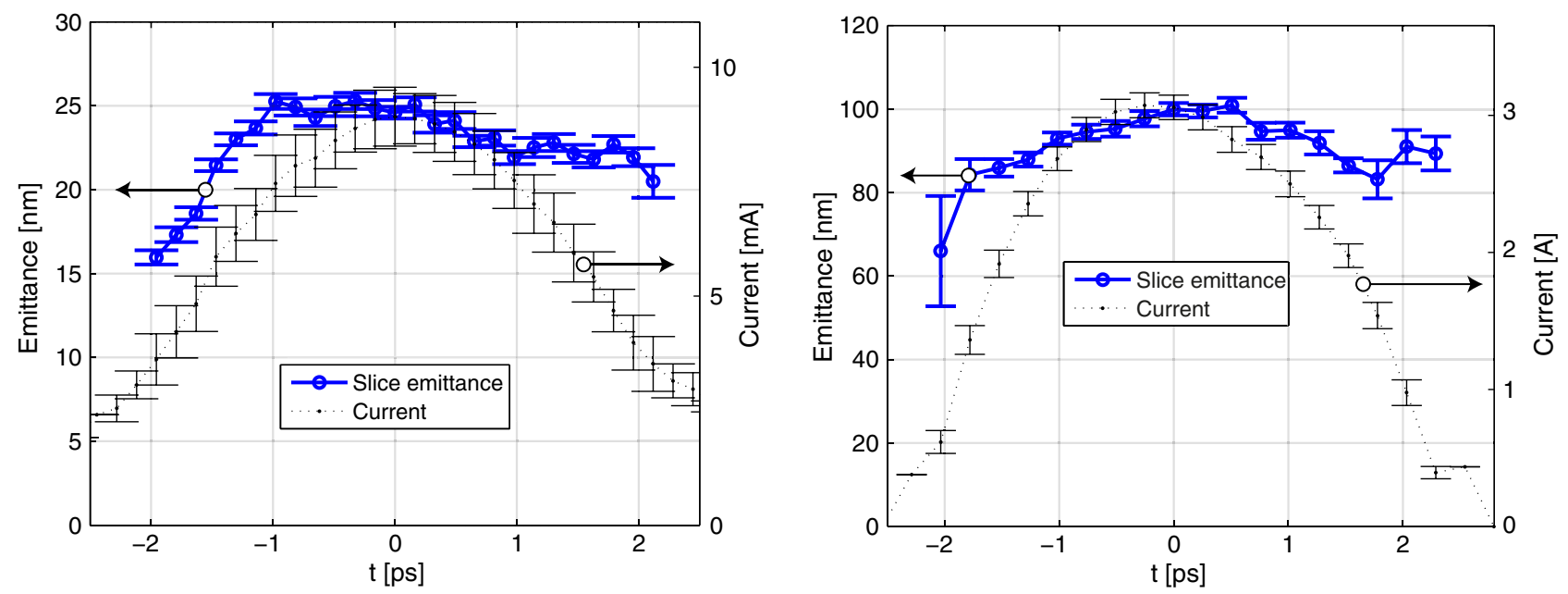

FIG. 7. Slice emittance measurement of a $30 \mathrm{fC}$ beam (left) and of a $10 \mathrm{pC}$ beam (right). The plot shows the charge distribution (black curve) as well as the emittance of the slices (blue curve).

dominant over other systematic errors such as energy uncertainties or quadrupole field errors - the magnitude of these two error sources is around $0.1 \ldots 0.2 \%$ according to the accuracy of beam momentum and magnetic field measurements done at the SwissFEL Injector Test Facility. The optics of the beam core were well matched to avoid a systematic increase of the error of the reconstructed parameters due to optics mismatch.

\section{CONCLUSION}

A transverse profile imager to measure the twodimensional profile of ionizing electron or photon beams has been built. The observation geometry of the scintillating crystal observes the Snell-Descartes law of refraction and the Scheimpflug imaging principle and thus results in a resolution better than $10 \mu \mathrm{m}$ across a field of view of $6 \times 14 \mathrm{~mm}^{2}$. This monitor was installed in the SwissFEL Injector Test Facility, and it has been used on a daily basis for measurements of beam profiles, projected and slice emittance. The resolution of the monitor is sufficient to measure a normalized slice emittance of $25 \mathrm{~nm}$ for a beam with a charge of $30 \mathrm{fC}$.

Different scintillators have been compared, and for uncompressed electron bunches, the measured beam image sizes using Ce:YAG were found to be comparable to measurements using optical transition radiation.

\section{ACKNOWLEDGMENTS}

The authors would like to express their sincere thanks to the many people at PSI who contributed to the design and construction of the profile monitor, including the mechanical engineering department, the vacuum and diagnostics sections. We are furthermore grateful to the SwissFEL commissioning team who operates the SwissFEL Injector Test Facility, and for support from the radiofrequency, laser, controls, alignment, magnet and infrastructure departments of PSI. Furthermore, we are grateful to the company Crytur for the scintillating crystal that is installed in the profile monitor.

[1] P. Emma et al., First lasing and operation of an ångstromwavelength free-electron laser, Nat. Photonics 4, 641 (2010).

[2] T. Ishikawa, M. Yabashi et al., A compact X-ray freeelectron laser emitting in the sub-ångström region, Nat. Photonics 6, 540 (2012).

[3] J. Madey, Stimulated emission of bremsstrahlung in a periodic magnetic field, J. Appl. Phys. 42, 1906 (1971).

[4] R. Akre et al., Commissioning the Linac Coherent Light Source injector, Phys. Rev. ST Accel. Beams 11, 030703 (2008).

[5] H. Loos et al., Observation of coherent optical transition radiation in the LCLS linac, in Proceedings of the 30th International Free-Electron Laser Conference, Gyeongju, Korea, 2008 (JACoW, Geneva, 2008), p. 485.

[6] A. H. Lumpkin et al., Evidence for transverse dependencies in COTR and microbunching in a SASE FEL, Nucl. Instrum. Methods Phys. Res., Sect. A 507, 200 (2003).

[7] A. Lumpkin, N. Sereno, W. J. Berg, M. Borland, Y. Li, and S. Pasky, Characterization and mitigation of coherentoptical-transition-radiation signals from a compressed electron beam, Phys. Rev. ST Accel. Beams 12, 080702 (2009).

[8] D. Ratner, Z. Huang, and G. Stupakov, Analysis of shot noise suppression for electron beams, Phys. Rev. ST Accel. Beams 14, 060710 (2011).

[9] D. Ratner, Ch. Behrens, Y. Ding, Z. Huang, A. Marinelli, T. J. Maxwell, and F. Zhou, Time-resolved imaging of the microbunching instability and energy spread at the Linac Coherent Light Source, Phys. Rev. ST Accel. Beams 18, 030704 (2015).

[10] Y. Otake et al., Commissioning and Performance of the Beam Monitor System for XFEL/SPring-8 SACLA, in Proceedings of the 2nd International Particle Accelerator 
Conference, San Sebastián, Spain, 2011 (JACoW, Geneva, 2011), p. 47.

[11] Ch. Wiebers, M. Holz, G. Kube, D. Nölle, G. Priebe, and H. Ch. Schröder, Scintillating screen monitors for transverse electron beam profile diagnostics at the European XFEL, in Proceedings of the 2nd International Beam Instrumentation Conference, Oxford, United Kingdom, 2013 (JACoW, Geneva, 2013), p. 807.

[12] T. Scheimpflug, Improved method and apparatus for the systematic alteration or distortion of plane pictures and images by means of lenses and mirrors for photography and for other purposes, GB Patent 1196 (1904).

[13] This law was actually described by Ibn Sahl in his work $O n$ Burning Mirros and Lenses in the year 984, long before the re-discovery by Snell and Descartes. See R. Rashed, A pioneer in anaclastics: Ibn Sahl on burning mirrors and lenses, Isis, 81, No. 3, p. 464, (1990).

[14] R. Ischebeck, J. Feldhaus, Ch. Gerth, and E. Saldin, Study of the transverse coherence at the TTF free electron laser, Nucl. Instrum. Methods Phys. Res., Sect. A 507, 175 (2003).

[15] A. Specka et al., High resolution beam monitoring with optical transition radiation at $3 \mathrm{MeV}$ electron energy, in Proceedings of the 15th International Particle Accelerator Conference, PAC-1993, Washington, DC, 1993 (IEEE, New York, 1993), p. 2450.

[16] B. X. Yang, J. L. Bailey, D. R. Walters, and S. J. Stein, Design of a high-resolution optical transition radiation imager for the linac coherent light source undulator, in Proceedings of the 21st International Particle Accelerator Conference, Knoxville, Tennessee, 2005 (IEEE, Piscataway, NJ, 2005), p. 4209.

[17] M. Pedrozzi, SwissFEL Injector Conceptual Design Report No. PSI-10-05, 2010.

[18] R. Ischebeck, E. Prat, V. Schlott, V. Thominet, M. Yan, P. Krejcik, and H. Loos, Transverse profile monitors for SwissFEL, in Proceedings of the 3rd International Beam Instrumentation Conference, Monterey, California, 2014 (JACoW, Geneva, 2014).

[19] B. Walasek-Höhne and G. Kube, Scintillating screen applications in beam diagnostics, in Proceedings of the 10th European Workshop on Beam Diagnostics and Instrumentation for Particle Accelerators, Hamburg, Germany, 2011 (JACoW, Geneva, 2011), p. 553.

[20] B. K. Scheidt, Upgrade of the ESRF fluorescent screen monitors, in Proceedings of the 6th European Workshop on Beam Diagnostics and Instrumentation for Particle Accelerators, Mainz, Germany, 2003 (JACoW, Geneva, 2003), p. 125.

[21] T. Kudo, S. Takahashi, N. Nariyama, T. Hirono, T. Tachibana, and H. Kitamura, Synchrotron radiation $\mathrm{X}$-ray beam profile monitor using chemical vapor deposition diamond film, Rev. Sci. Instrum. 77, 123105 (2006).

[22] R. Ischebeck, B. Beutner, B. Steffen, and V. Schlott, Screen monitor design for the SwissFEL, in Proceedings of the 9th European Workshop on Beam Diagnostics and Instrumentation for Particle Accelerators, Basel, Switzerland (JACoW, Geneva, 2009), p. 405.

[23] V. E. Scarpine, A. H. Lumpkin, W. Schappert, and G. R. Tassotto, Optical transition radiation imaging of intense proton beams at FNAL, IEEE Trans. Nucl. Sci. 51, 1529 (2004).
[24] W. S. Graves, E. D. Johnson, and P. G. O'Shea, A high resolution electron beam profile monitor, in Proceedings of the Particle Accelerator Conference, Vancouver, BC, Canada, 1997 (IEEE, New York, 1997), p. 1993.

[25] S. Matsubara, H. Maesaka, S. Inoue, and Y. Otake, Improvement of screen monitor with suppression of coherent-OTR effect for SACLA, in Proceedings of the 1st International Beam Instrumentation Conference, Tsukuba, Japan, 2012 (JACoW, Geneva, 2012), p. 34.

[26] A. Marinelli, M. Dunning, S. Weathersby, E. Hemsing, D. Xiang, G. Andonian, F. O'Shea, J. Miao, C. Hast, and J. B. Rosenzweig, Single-Shot Coherent Diffraction Imaging of Microbunched Relativistic Electron Beams for Free-Electron Laser Applications, Phys. Rev. Lett. 110, 094802 (2013).

[27] C. Behrens, Ch. Gerth, G. Kube, B. Schmidt, S. Wesch, and M. Yan, Electron beam profile imaging in the presence of coherent optical radiation effects, Phys. Rev. ST Accel. Beams 15, 062801 (2012).

[28] M. Yan, C. Behrens, Ch. Gerth, G. Kube, B. Schmidt, and S. Wesch, Suppression of coherent optical transition radiation in transverse beam diagnostics by utilising a scintillation screen with a fast gated CCD camera, Proceedings of the 10th European Workshop on Beam Diagnostics and Instrumentation for Particle Accelerators, Hamburg, Germany, 2011 (JACoW, Geneva, 2011), p. 440.

[29] S. O. Flyckt and C. Marmonier, Photomultiplier TubesPrinciples \& Applications, Photonis, Brive, France, 2002.

[30] M. Altarelli et al., Technical Report No. DESY 2006-097, 2007.

[31] J. Stohr et al., Technical Report No. SLAC-R-978, 2011.

[32] A. Lumpkin, A. S. Johnson, J. Ruan, J. Santucci, Y. E. Sun, and R. Thurman-Keup, Spatial Resolution Limits of YAG: Ce Powder Beam-Profile Monitors at the Fermilab A0 Photoinjector, Proceedings of the Sixth International Free Electron Laser Conference, Liverpool, UK (JACoW, Geneva, 2009), p. 348.

[33] G. Kube, C. Behrens, Ch. Gerth, B. Schmidt, M. Yan, and W. Lauth, Inorganic scintillators for particle beam profile diagnostics of highly brilliant and highly energetic electron beams, Proceedings of the 3rd International Particle Accelerator Conference, New Orleans, Lousiana (JACoW, Geneva, 2012), p. 2119.

[34] R. Ischebeck and V. Thominet, Transverse profile imager for ionizing radiation, European Patent EP 2700979 A1 (2014).

[35] G. Kube, Ch. Behrens, and W. Lauth, Resolution studies of inorganic scintillation screens for high energy and high brilliance electron beams, in Proceedings of the 1st International Particle Accelerator Conference, Kyoto, Japan, 2010 (JACoW, Geneva, 2010), p. 906.

[36] Zemax, LLC, Redmond, WA (USA).

[37] R. Ischebeck, B. Beutner, G. L. Orlandi, M. Pedrozzi, T. Schietinger, V. Schlott, and V. Thominet, Profile monitors for the SwissFEL injector test facility, in Proceedings of the 25th International Linear Accelerator Conference, Tsukuba, Japan, 2010 (JACoW, Geneva, 2010), p. 656.

[38] R. Ganter et al., SwissFELConceptual Design Report, No. PSI-10-04, 2012.

[39] Crytur Ltd., Turnov, Czech Republic.

[40] Diamond Materials GmbH, Freiburg, Germany. 
[41] Crytur Ltd., Turnov, Czech Republic.

[42] BCE Special Ceramics, Mannheim, Germany.

[43] LT Ultra, Herdwangen-Schoenach, Germany.

[44] Nikon, Tochigi, Japan.

[45] PCO AG, Kelheim, Germany.

[46] University Wafers, South Boston, MA, USA.

[47] Kodak, Rochester, NY, USA.
[48] M. Castellano and V. Verzilov, Spatial resolution in optical transition radiation beam diagnostics, Phys. Rev. ST Accel. Beams 1, 062801 (1998).

[49] E. Prat, M. Aiba, S. Bettoni, B. Beutner, S. Reiche, and T. Schietinger, Emittance measurements and minimization at the SwissFEL Injector Test Facility, Phys. Rev. ST Accel. Beams 17, 104401 (2014). 